\title{
A Comment on "Can Relaxation of Beliefs Rationalize the Winner's Curse?: An Experimental Study"*
}

\author{
Miguel A. Costa-Gomes ${ }^{\dagger} \quad$ Makoto Shimoji ${ }^{\ddagger}$ \\ First Version, December 24, 2010; This version, June 30, 2014
}

\begin{abstract}
Ivanov, Levin, and Niederle (2010) use a common-value second-price auction experiment to reject beliefs-based explanations for the winner's curse. ILN's conclusion however stems from the misuse of theoretical arguments. Beliefsbased models are even compatible with some observations from ILN's experiment.
\end{abstract}

\footnotetext{
${ }^{*}$ We thank Asen Ivanov and Muriel Niederle for sharing their data with us, and Asen Ivanov for providing a working paper version of the article and answering clarification questions. We thank the co-editor and anonymous referees for their thoughtful comments and suggestions which have improved the manuscript substantially. We thank Francesco Bravo, Nick Feltovich, Ron Harstad, Nagore Iriberri, Dan Knoepfle, John Nachbar, Tatsuyoshi Okimoto, Joel Sobel, and especially Vincent Crawford for advice and comments. We also thank the audience at the 2011 BIC Microeconomic Theory Workshop at the University of St. Andrews. Costa-Gomes thanks the hospitality of the Department of Economics and Related Studies at the University of York.

${ }^{\dagger}$ School of Economics and Finance, Castlecliffe, The Scores, University of St. Andrews, St. Andrews, UK, Email: miguel.costa-gomes@st-andrews.ac.uk

${ }^{\ddagger}$ Department of Economics and Related Studies, University of York, Heslington, York, YO10 5DD, UK, Email: makoto.shimoji@york.ac.uk
} 


\section{Introduction}

Ivanov, Levin, and Niederle (2010, ILN henceforth) claim that the results from their common-value second-price auction experiment casts a doubt on beliefs-based models as explanations for the winner's curse. In this note, we first argue that ILN's theoretical arguments are misleading (Section 2). We then show that beliefs-based models do not necessarily imply ILN's predictions and are compatible with some observations (Sections 3 and 4). We also discuss other points (Section 5).

\section{ILN's Experiment and Theoretical Arguments}

We first describe ILN's study. ILN's experiment considers a common-value secondprice auction with two bidders, called the maximal game. In this auction, each bidder receives a private signal $X_{i}(i=1,2)$, uniformly distributed over $X=\{0,1, \ldots, 10\}$ $(|X|=11)$. The value of the item is given by $x^{\max }=\max \left\{x_{1}, x_{2}\right\} \quad\left(X^{\max }=\right.$ $\max \left\{X_{1}, X_{2}\right\}$ ) where $x_{i}$ is the realization of $X_{i}$. Each player's bid is chosen from the set $B=\{0,0.01,0.02, \ldots, 1000000.00\}(|B|=100000001)$. We use "max" to refer to the highest possible bid, 1000000.00. A player's strategy is a map $b_{i}: X \rightarrow B$. The bidder with the highest bid wins and pays the second highest bid. Ties are broken with equal probabilities. We assume that players' payoff functions only depend on the monetary outcome and that players are risk neutral. ${ }^{1}$

ILN's experiment has three treatments: Baseline, ShowBidFn, and MinBid (BL, $S B F$, and $M B$ henceforth). Each treatment has two parts, I and II, each of which consists of 11 auctions. In each auction of part I, a subject receives a different signal and plays against a randomly selected subject. The former allows us to observe the pure strategy each subject chose in part I. In part II, a subject plays against a computer which receives an i.i.d. signal from $X$ and plays the subject's pure strategy from part I. ${ }^{2} S B F$ and $M B$ are variants of $B L$; (i) each subject is explicitly shown the pure strategy for the computer in part II (i.e., her part I bids) in $S B F$, and (ii) the set of bids is rather $\left\{x_{i}, x_{i}+0.01, \ldots, \max \right\}$ for each $x_{i} \in X$ in $M B$.

ILN focus on what they call "overbidding", $b_{i}\left(x_{i}\right) \in\left(x_{i}, 10\right]$, and present the

\footnotetext{
${ }^{1}$ Although it is not explicitly stated, the proof of Proposition 3 in ILN, for example, suggests that ILN also assume risk neutrality.

${ }^{2}$ Subjects are only informed of these features after part I.
} 
following two predictions: ${ }^{3}$

Q1 “... if behavior is driven by beliefs, we should observe a reduction in overbidding (i) in part II of each treatment relative to part I and (ii) in part I of the MinBid treatment relative to part I of the Baseline and ShowBidFn treatments. The absence of any such reduction would cast serious doubt on belief-based theories." (p.1440)

ILN claim that their experiment shows no evidence supporting Q1 and reject analogybased expectation equilibrium (Jehiel (2005) and Jehiel and Koessler (2008), ABEE henceforth), cursed equilibrium (Eyster and Rabin (2005), CE henceforth), and level$k$ reasoning (Crawford and Iriberri (2007)) for the winner's curse. ILN (footnote 4) indeed claim that their study applies to any beliefs-based explanation of the winner's curse.

We now document ILN's reasoning for Q1. Regarding Q1 (i), ILN state:

Q2 "Consider a subject $i$ who overbids (for all signals) in part I of one of the three treatments. From Proposition 1, it follows that bidding her signal is a best response in part II. ... if $i$ continues overbidding without a downward correction or even starts bidding above 10 in part II, she is clearly not best responding to her behavior from part I." (pp.1440-1441)

Proposition 1 in ILN shows that $b_{i}\left(x_{i}\right)=x_{i}$ for each $x_{i} \in X$ is the only bid that survives two rounds of iterated weak dominance. Regarding Q1 (ii), ILN state:

Q3 "In part I of the MinBid treatment, anything other than bidding one's signal is weakly dominated." (p.1441)

Note that both arguments refer to weak dominance. They are misleading, however.

Regarding Q2, the analysis of part II requires no game theoretical argument. Remember that each subject faces a computer as her opponent in part II which mimics the subject's behavior in part I. That is, each subject knows her opponent's strategy in part II. Thus, weak dominance necessarily corresponds to expected payoff

\footnotetext{
${ }^{3}$ For their focus on overbidding, ILN (p.1440) state that " $(\mathrm{t})$ he most interesting behavior is overbidding because it leads to a WC (as long as others are also appropriately overbidding) and because it could potentially be explained by belief-based theories." Overbidding is not sufficient for the winner's curse; e.g., if $b_{i}\left(x_{i}\right) \in\left(x_{i}, x_{i}+1\right)$ for each $i$ and $x_{i} \in X \backslash\{10\}$ and $b_{i}(10)=10$, the winner's curse does not arise for higher signals.
} 
maximization (i.e., single-person decision problem) in part II. This also suggests that the comparison of parts I and II is irrelevant. In Section 3, we use the data from ILN's experiment and show that expected payoff maximization does not necessarily imply "downward correction" and may even be consistent with bidding above $10 .{ }^{4}$

Regarding Q3, ABEE, CE, and level- $k$ reasoning mentioned above rather assume that players best-respond to their beliefs. It is known that weak dominance and best response (strict dominance) may provide distinct predictions since a best response strategy can be weakly dominated. ${ }^{5}$ To discuss ABEE, CE, and level- $k$ reasoning, referring to weak dominance is simply not appropriate. We show in Section 4 that this distinction is crucial for the comparison of parts $\mathrm{I}$ in $B L / S B F$ and $M B$.

\section{Analysis of Part II}

Auctions in part II are single-person decision problems. Expected payoff maximization implies "downward corrections" in part II if $b_{i}\left(x_{i}\right)>x_{i}$ for each $x_{i} \in X$ in part I - this is what ILN state in the parentheses in Q2. The data from ILN's experiment shows that it need not be the case otherwise. We select three subjects from their experiment; \#37 $(B L)$, \#133 $(S B F)$, and \#114 $(M B) .{ }^{6}$ For each of them, Table 1 lists the part I bid (PI) and the set of expected payoff maximizing bids in part II (PMB) for each signal. Note (i) that they could choose higher bids - even "max" - in part II than in part I for 6 (\#37), 7 (\#133) and 5 (\#114) signals to maximize their expected payoffs (denoted with a "*") and (ii) that $b_{i}\left(x_{i}\right)=x_{i}$ does not necessarily maximize a subject's expected payoffs in part II of $B L / S B F$ (denoted with a " $\nmid$ ").

We now turn to ILN's data analysis. ILN use a set of criteria to select subjects. Each bid is placed into one of four categories; (i) $b_{i}\left(x_{i}\right)<x_{i}-0.25$, (ii) $b_{i}\left(x_{i}\right) \in$ $\left[x_{i}-0.25, x_{i}+0.25\right]$, (iii) $b_{i}\left(x_{i}\right) \in\left(x_{i}+0.25,10\right]$, and (iv) $b_{i}\left(x_{i}\right)>10 .{ }^{7}$ For each part, each subject is classified as either (i) Underbidder, (ii) Signal Bidder, (iii) Overbidder, or (iv) Above-10 Bidder if the majority of her bids (6 or more out of 11) fall into

\footnotetext{
${ }^{4}$ Proposition 1 in ILN uses iterated weak dominance. Best-responding to the opponent's specific strategy does not necessarily imply $b_{i}\left(x_{i}\right)=x_{i}$ for each $x_{i} \in X$. This also shows that referring to Proposition 1 is not appropriate.

${ }^{5}$ In $M B$, for example, while $b_{i}\left(x_{i}\right)=x_{i}$ is the weakly dominant strategy for each $x_{i} \in X$, for each strategy, there exists a belief to which the strategy in concern is a best response.

${ }^{6}$ They choose $b_{i}\left(x_{i}\right) \in\left(x_{i}+0.25,10\right]$ for six signals in part I (emphasized in bold face) and are Overbidders in part I according to ILN's classification.

${ }^{7}$ For $x_{i}=10$, (iii) is omitted and (iv) is replaced with $b_{i}(10)>10.25$.
} 


\begin{tabular}{|c||c|c||c|c||c|c|}
\hline \multicolumn{1}{|c||}{} & \multicolumn{2}{c||}{$\# 37(B L)$} & \multicolumn{2}{c|}{$\# 133(S B F)$} & \multicolumn{2}{c|}{$\# 114(M B)$} \\
\cline { 2 - 7 }$x_{i}$ & PI & PMB & PI & PMB & PI & PMB \\
\hline \hline 0 & $\mathbf{4}$ & $\{0, \ldots, 3.99\}$ & 0 & $\{4.03, \ldots, 5.01\}^{*, \dagger}$ & 0 & $\{0, \ldots, 5.99\}^{*}$ \\
\hline 1 & 1 & $\{0, \ldots, 3.99\}^{*}$ & $\mathbf{3 . 0 1}$ & $\{4.03, \ldots, 5.01\}^{*, \dagger}$ & $\mathbf{6}$ & $\{1, \ldots, 5.99\}$ \\
\hline 2 & $\mathbf{4}$ & $\{1.01, \ldots, 3.99\}$ & $\mathbf{4}$ & $\{4.03, \ldots, 5.01\}^{*, \dagger}$ & 2 & $\{2, \ldots, 5.99\}^{*}$ \\
\hline 3 & $\mathbf{5}$ & $\{1.01, \ldots, 3.99\}$ & $\mathbf{6}$ & $\{4.03, \ldots, 5.01\}^{\dagger}$ & 3 & $\{3, \ldots, 5.99\}^{*}$ \\
\hline 4 & $\mathbf{5}$ & $\{1.01, \ldots, 4.99\} \cup\{6.01, \ldots, 8.99\}^{*}$ & $\mathbf{5 . 0 2}$ & $\{4.03, \ldots, 5.01\}^{\dagger}$ & $\mathbf{6}$ & $\{4, \ldots, 5.99\}$ \\
\hline 5 & 5 & $\{6.01, \ldots, 8.99\}^{* \dagger}$ & 4.02 & $\{6.01, \ldots, 8\}^{*, \dagger}$ & $\mathbf{6}$ & $\{5, \ldots, 5.99\}$ \\
\hline 6 & 5 & $\{6.01, \ldots, 8.99\}^{* \dagger}$ & $\mathbf{8 . 0 1}$ & $\{6.01, \ldots, 8\}^{\dagger}$ & $\mathbf{8}$ & $\{6, \ldots, 7.99\}$ \\
\hline 7 & 6 & $\{6.01, \ldots, 8.99\}^{*}$ & $\mathbf{9}$ & $\{8.02, \ldots, 8.99\}^{\dagger}$ & $\mathbf{9}$ & $\{7, \ldots, 7.99\}$ \\
\hline 8 & $\mathbf{9}$ & $\{6.01, \ldots, 8.99\}$ & 6 & $\{8.02, \ldots, 8.99\}^{*, \dagger}$ & 8 & $\{8, \ldots, 8.99\}^{*}$ \\
\hline 9 & $\mathbf{1 0}$ & $\{6.01, \ldots, 9.99\}$ & 4 & $\left\{8.02, \ldots, \max ^{*}\right.$ & $\mathbf{1 0}$ & $\{9, \ldots, 9.99\}$ \\
\hline 10 & 10 & $\left\{9.01, \ldots, \max ^{*}\right.$ & 8.01 & $\left\{9.01, \ldots, \max ^{*}\right.$ & 10 & $\left\{10, \ldots, \max ^{*}\right.$ \\
\hline
\end{tabular}

Table 1: Three subjects from ILN's Experiment

\begin{tabular}{|c||c|c|c||c|}
\hline & $B L$ & $S B F$ & $M B$ & All Treatments \\
\hline \hline Number of Subjects & 62 & 46 & 26 & 134 \\
\hline \hline Overbidder I & $25(40.3 \%)$ & $18(39.1 \%)$ & $19(73.1 \%)$ & $62(46.3 \%)$ \\
\hline Overbidder IEIII & $14(22.6 \%)$ & $10(21.7 \%)$ & $14(53.9 \%)$ & $38(28.4 \%)$ \\
\hline \hline Above-Signal Bidder I & $44(71.0 \%)$ & $32(69.6 \%)$ & $22(88.6 \%)$ & $98(73.1 \%)$ \\
\hline
\end{tabular}

Table 2: Classification

one of these four categories. ${ }^{8}$ According to this classification, 62 out of 134 subjects $(46.3 \%)$ are Overbidders in part I (Overbidder I). Instead of focusing on their behavior to examine the presence of "downward corrections" in part II, ILN restrict their analysis to 38 subjects (28.4\%) who are Overbidders in both parts (Overbidder IEII). The details are included in Table 2. Given the set of these subjects, ILN computed the median bid for each signal, each treatment, and each part. Figures 1, 4 and 5 in ILN plot them and show that the numbers of signals for which the median is lower in part I than in part II are only $2(B L), 3(S B F)$, and $1(M B)$. ILN (Result $2(\mathrm{~b})$ ) conclude that there is no evidence of a downward correction of the bids.

As shown above, the examination of downward corrections is irrelevant. Given the structure of part II, while attempting to maintain ILN's focus on Overbidders, we examine subjects' behavior in part II with some modifications:

\footnotetext{
${ }^{8}$ Otherwise, the subject is classified as Indeterminate. There is no Underbidder category in $M B$.
} 
1. We do not use subjects' part II bids as a selection criterion - we (as well as ILN) analyze subjects' behavior in part II. We rather focus on 98 subjects, referred as Above-Signal Bidder I; for each of them, the majority of her bids (at least 6 out of 11) are higher than the corresponding signals in part I. ${ }^{9}$ Table 2 contains the details.

2. We do not compare the median bids in parts I and II. Instead, we use a statistical test to compare subjects' part II median bid with the median of the subjects' upper bound of the set of expected payoff maximizing bids in part II. We use Fisher-Pitman's permutation test for paired-samples in order to account for the fact that a subject's upper bound of the set of expected payoff maximizing bids in part II is related to her bids in the part I auctions. We test the null hypothesis that the median of the distribution of part II bids is smaller than or equal to the median of the distribution of the upper bound of the set of expected payoff maximizing bids in part II against the alternative that it is larger.

Using a significance level of $5 \%$, the Fisher-Pitman permutation test for paired samples rejects the null for 4 out of 11 signals in each of the treatments - 0, 1, 2 and 3 in $B L, 0,1,2$ and 4 in $S B F$, and 2, 4, 5 and 7 in $M B$. Therefore, the test tells us that for 7 of the signals the median part II bid is not greater than the corresponding upper bound of the set of the expected payoff maximizing part II bids. Therefore, subjects are not overbidding in relation to their optimal bids for most of the signals in all three treatments. These results are at odds with ILN's conclusion. ${ }^{10}$

\section{Comparison of Parts I in $B L / S B F$ and $M B$}

ILN computed the average of the bids with $b_{i}\left(x_{i}\right) \in\left(x_{i}+0.25,10\right]$ for each signal in parts I of $B L / S B F$ and $M B$. While ILN's prediction suggests that the bids are

\footnotetext{
${ }^{9}$ We do not exclude bids above 10 and do not use ILN's 0.25 tolerance level. For the latter, ILN (footnote 28) claim that it does not affect their conclusion. Above-Signal Bidder I has $19(B L), 14$ $(S B F)$ and $3(M B)$ more subjects than Overbidder I. The inclusion of bids above 10 adds 17 ( $B L$; one of which would also be added by dropping the tolerance level), $12(S F B)$, and $3(M B)$ more subjects.

${ }^{10}$ One can focus on the subjects with $b_{i}\left(x_{i}\right)>x_{i}$ for each $x_{i} \in X$, to whom "for all signals" in Q2 applies. There are $12(19.4 \%)$ in $B L, 4(8.7 \%)$ in $S B F$, and $5(19.2 \%)$ in $M B$. The medians are indeed lower in part II than in part $I$ for all signals $(B L), 9$ signals $(S B F)$, and 8 signals $(M B)$, implying "downward corrections" for most signals.
} 


\begin{tabular}{|c||c|c||c|c|}
\hline \multicolumn{1}{|c||}{} & \multicolumn{2}{c||}{$B L / S B F$} & \multicolumn{2}{c|}{$M B$} \\
\cline { 2 - 5 }$x_{i}$ & $L 1$ & $L 2$ & $L 1$ & L2 \\
\hline \hline 0 & 5 & $\{0, \ldots, 4.99\}$ & 0 & $\{0, \ldots, 10\}$ \\
\hline 1 & 5.09 & $\{0, \ldots, 4.99\}$ & 1 & $\{1, \ldots, 10\}$ \\
\hline 2 & 5.27 & $\{0, \ldots, 4.99\}$ & 2 & $\{2, \ldots, 10\}$ \\
\hline 3 & 5.55 & $\{0, \ldots, 4.99\}$ & 3 & $\{3, \ldots, 10\}$ \\
\hline 4 & 5.91 & $\{0, \ldots, 4.99\}$ & 4 & $\{4, \ldots, 10\}$ \\
\hline 5 & 6.36 & $\{0, \ldots, 5.08\}$ & 5 & $\{5, \ldots, 10\}$ \\
\hline 6 & 6.91 & $\{5.92, \ldots, 6.35\}$ & 6 & $\{6, \ldots, 10\}$ \\
\hline 7 & 7.55 & $\{6.92, \ldots, 7.54\}$ & 7 & $\{7, \ldots, 10\}$ \\
\hline 8 & 8.27 & $\{7.56, \ldots, 8.26\}$ & 8 & $\{8, \ldots, 10\}$ \\
\hline 9 & 9.09 & $\{8.28, \ldots, 9.08\}$ & 9 & $\{9, \ldots, 10\}$ \\
\hline 10 & $\{10, \ldots, \max \}$ & $\{9.10, \ldots, 10\}$ & $\{10, \ldots, \max \}$ & 10 \\
\hline
\end{tabular}

Table 3: Bid Correspondences for $L 1$ and $L 2$

lower in $M B$ than $B L / S B F$, the comparison of these averages show that they are "astonishingly close" (ILN p.1445). In this section, we show that beliefs-based models do not necessarily imply their predictions and are compatible with this observation.

We first focus on the level- $k$ model which incorporates players' finite depth of reasoning. We (as well as ILN) focus on random level- $k$ (henceforth $L k$ ) players who best-respond to level- $(k-1)$ players for $k \in\{1,2, \ldots\}$ and anchor their beliefs in a $L 0$ player who chooses her bid with equal probability from $\{0,0.01, \ldots, 10\}$ in $B L / S B F$ and $\left\{x_{i}, x_{i}+0.01, \ldots, 10\right\}$ in $M B$. Table 3 shows $L 1$ 's and $L 2$ 's bid correspondences in each treatment. ${ }^{11}$ While $L 1$ 's bid functions are consistent with Q1 (ii), ILN's experimental setting allows $L 2$ to have wide ranges of bids with which ILN's prediction cannot be uniquely deduced. ${ }^{12}$ Note also that $L 2$ 's behavior is compatible with $b_{i}\left(x_{i}\right)>x_{i}$ for each $x_{i} \in X \backslash\{10\}$ and similar behavior in all treatments.

ABEE captures the idea that players bundle states into analogy classes and bestrespond to beliefs which average the opponent's strategy within each class. CE assumes that players best-respond to beliefs which assign $\chi \in[0,1]$ to the opponent's "average" strategy and $1-\chi$ to the opponent's type-dependent strategy. If we use (i) the private information analogy partition for ABEE and (ii) $\chi=1$ (fully cursed)

\footnotetext{
${ }^{11} L 1$ 's bids in $B L / S B F$ are either $E\left[X^{\max } \mid x_{i}\right]+1$ or $E\left[X^{\max } \mid x_{i}\right]-1$ for each $x_{i} \in X \backslash\{10\}$. See also Proposition 3 in ILN. Table 4 lists the values of $E\left[X^{\max } \mid x_{i}\right]$ for each $x_{i} \in X$.

${ }^{12}$ Crawford and Iriberri (2007) show that a mixture of $L 1$ s and $L 2$ s explains auction data well.
} 


\begin{tabular}{|c||c||c|c||c|c|}
\hline$x_{i}$ & $E\left[X^{\max } \mid x_{i}\right]$ & $b^{l}\left(x_{i}\right)$ & $\rho\left(x_{i}\right)$ & $b^{h}\left(x_{i}\right)$ & $1-\rho\left(x_{i}\right)$ \\
\hline \hline 0 & 5 & 5 & - & 5 & - \\
\hline 1 & $\frac{56}{11} \approx 5.091$ & 5.09 & $\frac{10}{11}$ & 5.10 & $\frac{1}{11}$ \\
\hline 2 & $\frac{58}{11} \approx 5.273$ & 5.27 & $\frac{8}{11}$ & 5.28 & $\frac{3}{11}$ \\
\hline 3 & $\frac{61}{11} \approx 5.545$ & 5.54 & $\frac{5}{11}$ & 5.55 & $\frac{6}{11}$ \\
\hline 4 & $\frac{65}{11} \approx 5.909$ & 5.90 & $\frac{1}{11}$ & 5.91 & $\frac{10}{11}$ \\
\hline 5 & $\frac{70}{11} \approx 6.364$ & 6.36 & $\frac{7}{11}$ & 6.37 & $\frac{4}{11}$ \\
\hline 6 & $\frac{76}{11} \approx 6.909$ & 6.90 & $\frac{1}{11}$ & 6.91 & $\frac{10}{11}$ \\
\hline 7 & $\frac{83}{11} \approx 7.545$ & 7.54 & $\frac{5}{11}$ & 7.55 & $\frac{6}{11}$ \\
\hline 8 & $\frac{91}{11} \approx 8.273$ & 8.27 & $\frac{8}{11}$ & 8.28 & $\frac{3}{11}$ \\
\hline 9 & $\frac{100}{11} \approx 9.091$ & 9.09 & $\frac{10}{11}$ & 9.10 & $\frac{1}{11}$ \\
\hline 10 & 10 & 10 & - & 10 & - \\
\hline
\end{tabular}

Table 4: Equilibrium Strategy for ABEE and CE

for $\mathrm{CE}$, they coincide. ${ }^{13}$ As an example, we use these specifications for part I of $B L / S B F$. Table 4 shows an equilibrium strategy. ${ }^{14}$ The second column in Table 4 contains the expected value of the object given the signal. The third to the sixth columns specify each player's strategy: given signal $x_{i}$, the player bids either $b^{l}\left(x_{i}\right)$ with probability $\rho\left(x_{i}\right)$, or $b^{h}\left(x_{i}\right)$ with probability $1-\rho\left(x_{i}\right)$. For each $x_{i} \in X$, note (i) $b_{i}^{l}\left(x_{i}\right)<E\left[X^{\max } \mid x_{i}\right]<b_{i}^{h}\left(x_{i}\right)$ and (ii) $E\left[X^{\max } \mid x_{i}\right]=b_{i}^{l}\left(x_{i}\right) \rho\left(x_{i}\right)+b_{i}^{h}\left(x_{i}\right)\left(1-\rho\left(x_{i}\right)\right)$. In this equilibrium, each player chooses $b_{i}\left(x_{i}\right)>x_{i}$ for every $x_{i} \in X \backslash\{10\} .{ }^{15}$

To apply $\mathrm{ABEE}$ and $\mathrm{CE}$ to $M B$, we need to take into account that players' action spaces are type-dependent in $M B \cdot{ }^{16} \mathrm{ABEE}$ and $\mathrm{CE}$ assume that players' action spaces are fixed so that average behavior is well defined. Even if the original definitions are modified in this regard, the type-dependence limits the specifications. $M B$ only allows $\chi=0$ for $\mathrm{CE}$, which coincides with one of two possibilities for ABEE, i.e., the standard private information setting. ${ }^{17}$ In this case, they also coincide with Bayesian Nash Equilibrium (BNE henceforth). While Proposition 2 in ILN shows

\footnotetext{
${ }^{13}$ Jehiel and Koessler (2008, p.539) and Eyster and Rabin (2005, p.1634).

${ }^{14}$ See also Proposition 4 of ILN, which is based on Proposition 5 of Eyster and Rabin (2005).

${ }^{15}$ Any $b_{i}\left(x_{i}\right)<x_{i}$ is "underbidding" for ILN's theoretical argument. ILN (p.1440) state "to explain overbidding, both the level- $k$ model and CE require that beliefs place a positive weight on underbidding". $L 2$ in $B L / S B F$ and $\mathrm{ABEE} / \mathrm{CE}$ above serve as counter-examples.

${ }^{16}$ ILN (footnote 24) acknowledge this for CE.

${ }^{17}$ Any partition finer than the standard private information setting is also allowed. Since player $i$ 's strategy is defined with respect to the private information player $i$ has (as in BNE), the opponent's strategy is identical within each element of such finer partitions, which implies that any ABEE with a finer partition is also a BNE.
} 
that the unique symmetric BNE in $M B$ is $b_{i}\left(x_{i}\right)=x_{i}$ for each $x_{i} \in X$, there are other asymmetric BNE. ${ }^{18}$ The observations of $b_{i}\left(x_{i}\right)>x_{i}$ and similar behavior in all treatments could be explained by coordination failures. ${ }^{19}$ The other possibility for ABEE is the coarsest analogy partition which only includes the set of all states. ${ }^{20}$ In this case, the equilibrium strategy shown in Table 4 is also ABEE for both $B L / S B F$ and $M B$, implying $b_{i}\left(x_{i}\right)>x_{i}$ for each $X \backslash\{10\}$ and similar behavior in all treatments.

\section{Discussion}

To further analyze subjects' behavior in part II, Table 5 displays a relationship between the monotonicity of part I bids and the number of expected payoff maximizing bids in part II for each treatment. The second column shows the average number of expected payoff maximizing bids in part II. ${ }^{21}$ The third column shows the number of subjects who exhibit part I weakly monotone bidding behavior and the fifth column shows the number of the rest. The fourth and sixth columns show the average numbers of expected payoff maximizing bids. Two common observations to all treatments; (i) a small fraction of subjects exhibit bids increasing in signals, and (ii) the average number of expected payoff maximizing bids for such subjects is larger than that of the rest. ${ }^{22}$

We assume that players best-respond. Camerer, Nunnari and Palfrey (2011) relax

\footnotetext{
${ }^{18}$ For example, given $\alpha \in X$, player $i$ chooses $b_{i}\left(x_{i}\right) \in\left\{x_{i}, \ldots, \alpha\right\}$ for $x_{i} \in\{0, \ldots, \alpha\}$ and $b_{i}\left(x_{i}\right)=$ $x_{i}$ for $x_{i} \in\{\alpha+1, \ldots, 10\}$, and player $j$ chooses $b_{j}\left(x_{j}\right)=x_{j}$ for $x_{j} \in\{0, \ldots, \alpha\}$ and $b_{j}\left(x_{j}\right) \geq x_{j}$ for $x_{j} \in\{\alpha+1, \ldots, 10\}$. While acknowledging this, ILN (footnote 10) dismiss the possibility of asymmetric BNE.

${ }^{19}$ Indeed, every bid is (interim) rationalizable in the maximal game.

${ }^{20}$ Jehiel and Koessler (2008, p.538)

${ }^{21}$ This also shows that $S B F$ has the lowest average number of expected payoff maximizing bids, implying that being explicitly informed of the opponent's strategy did not help. A one-sided FlignerPolicello robust rank order test rejects (with a p-value of 0.035) the null that the distribution of subjects' number of expected payoff maximizing bids is the same in $B L$ and $S B F$. One plausible explanation for this is that it is difficult to fully incorporate all the informational details into the decision process. This may also explain similar behavior in parts I of $B L / S B F$ and $M B$.

${ }^{22} \mathrm{~A}$ Fligner-Policello robust rank order test rejects the null hypothesis that the empirical distributions of the number of expected payoff maximizing bids of the 22 subjects whose bidding behavior in part I is monotone and of the other 112 subjects come from the same distribution yields a $p$-value of 0.00. In addition, the closer a subject's bidding behavior in part I is to being monotone, the larger her number of expected payoff maximizing bids; an OLS regression of a subject's number of expected payoff maximizing bids on her number of pairs of weakly monotone adjacent bids in part I (i.e., for two consecutive signals) yields a positive coefficient for the independent variable (1.08, $p$-value of 0.000$)$.
} 


\begin{tabular}{|c||c||c|c||c|c|}
\hline \multicolumn{1}{|c||}{} & \multicolumn{1}{c||}{} & \multicolumn{2}{c||}{ Monotone } & \multicolumn{2}{c|}{ Non-monotone } \\
\cline { 2 - 6 } & PMB & Subjects & PMB & Subjects & PMB \\
\hline \hline$B L$ & 4.18 & 9 & 7.33 & 53 & 3.64 \\
& {$[38.0 \%]$} & $(14.5 \%)$ & {$[66.7 \%]$} & $(85.5 \%)$ & {$[33.1 \%]$} \\
\hline \multirow{2}{*}{$S B F$} & 3.07 & 7 & 6.86 & 39 & 2.38 \\
& {$[27.9 \%]$} & $(15.2 \%)$ & {$[62.3 \%]$} & $(84.8 \%)$ & {$[21.7 \%]$} \\
\hline \multirow{2}{*}{$M B$} & 4.73 & 6 & 8.83 & 20 & 3.50 \\
& {$[43.0 \%]$} & $(23.1 \%)$ & {$[80.3 \%]$} & $(76.9 \%)$ & {$[31.8 \%]$} \\
\hline
\end{tabular}

Table 5: Monotonicity

this assumption while adopting a structural approach to study how close subjects' bids are to their best responses by relaxing the assumption that subjects best-respond. Their analysis shows (i) that imperfect best response versions of beliefs-based models such as the Logit QRE, Cursed Equilibrium, and Cognitive Hierarchy fit the part I data well, and (ii) that the fitted parameters forecast part II behavior accurately.

\section{References}

[1] Camerer, C. F., S. Nunnari, and T. R. Palfrey (2011): "Quantal Response and Nonequilibrium Beliefs Explain Overbidding in Maximum-Value Auctions," Caltech Social Science Working Paper 1349.

[2] Crawford, V. P., and N. Iriberri (2007): "Level- $k$ Auctions: Can a NonEquilibrium Model of Strategic Thinking Explain the Winner's Curse and Overbidding in Private-Value Auctions?" Econometrica, 75, 1721-1779.

[3] Eyster, E., and M. Rabin (2005): "Cursed Equilibrium," Econometrica, 73, $1623-1672$.

[4] Ivanov, A., D. Levin, and M. Niederle (2010): "Can Relaxation of Beliefs Rationalize the Winner's Curse?: An Experimental Study," Econometrica, 78, 14351452.

[5] Jehiel, P. (2005): "Analogy-Based Expectation Equilibrium," Journal of Economic Theory, 123, 81-104. 
[6] Jehiel, P., and F. Koessler (2008): "Revisiting Games of Incomplete Information with Analogy-Based Expectations," Games and Economic Behavior, 62, 533557. 